\title{
Research on Intelligent Home System based on Wireless Sensor Network
}

\author{
Xiaodong Zhang ${ }^{1,}$, Wenshuai $\mathrm{Ji}^{1, \mathrm{~b}}$ \\ ${ }^{1}$ Henan Mechanical and Electrical Vocational College, Zhenzhou, Henan, 451191 \\ ${ }^{a}$ email, ${ }^{b}$ email
}

Keywords: Intelligent Home System, Wireless Sensor Network, IT

\begin{abstract}
At present, the existing smart home subsystem uses different hardware and software and communication protocol. It has a single function and does not have a remote intelligent terminal and some of the traditional smart home system uses wired network set up, they have maintenance problems and poor system scalability. So it is difficult to form a true sense of the intelligent home system, it is difficult to achieve a unified remote monitoring and centralized monitoring. Smart home system based on wireless sensor network can make up the above shortcomings.
\end{abstract}

\section{Introduction}

Smart home purpose is mainly for residential, and the application of a lot of existing technologies, such as a variety of different functions of the sensor, the relevant electronic equipment, control technology, security technology, and the use of communication technology to home electronic equipment As much as possible to connect a piece to the comfort of the components for the purpose of the other, he also can be more efficient unified management of family affairs, so its introduction leads to comfort, convenience, safety, efficiency greatly improved. Smart home is intelligent home. In fact, the process of developing intelligent home system is integrated smart home. Smart home, also known as smart home (Smart Home), many people also called smart home automation, digital home, home network and home networks, etc. At present, there are many mature and widely used examples of smart home, such as a variety of energy billing system, as a common variety of remote meter reading is based on the realization of it; most of our current district a wide range of measurement Table meter reading is still used on-site meter reading the way, obviously inefficient. The so-called smart remote meter reading system on the one hand can greatly improve the efficiency of meter reading, while human and material resources have also been released, so it has a positive significance.

\section{Wireless Home Networking Technology of Smart Home Systems}

Wireless transmission mainly includes Bluetooth technology, infrared technology, radio frequency technology and the rise of recent years, Wi-Fi and ZigBee communication technology.

Bluetooth technology features not only support asynchronous communication also supports synchronous communication, but also supports the circuit and packet switching features. As a result of these features, he allows simultaneous voice channels up to three simultaneously, but also supports the completion of a single channel asynchronous data transmission and synchronization of voice. The rate of any one of the synchronous voice channels is $64 \mathrm{k}$ bits per second. Asynchronous channel for ADSL-like asymmetric connection, that side of the rate of up to $721 \mathrm{k}$ bit per second, while the other side of the rate of only $57.6 \mathrm{k}$ bit per second, but he also supports symmetric connection, symmetrical connection rate of $43.2 \mathrm{kbps}$.

Infrared is a wavelength between $750 \mathrm{~nm}$ to $1 \mathrm{~mm}$ between the electromagnetic waves, its frequency is higher than the microwave and lower than the visible light, is a human eye can not see the light. In the wireless Internet access, as long as the phone with an infrared interface, configure the infrared connection agreement, you can not connect directly to the Internet can be achieved, so convenient networking. Many software and hardware platforms support this infrared interface, the interface has become a wireless connection technology, has been widely used. 
Radio frequency technology is a close-range, low complexity, low power, low data rate, low-cost wireless communication technology, the use of radio frequency signals through space coupling to achieve non-contact information transmission and automatic transmission of information through the identification purpose. Radio frequency technology has been widely used in industrial automation, business automation and home automation and other fields, which has a strong advantage to improve the efficiency of information processing and accuracy. Siemens, Motorola and Compaq Computer and other multinational companies to establish a radio frequency technology based on wireless networking standards, they set up the HameRF working group, which is responsible for research and development, their initial purpose is to develop a home network for the convenience of network, Use, lower cost of global standards. HomeRF working group to develop the standard, including voice and data communications, the use of the frequency of $10 \mathrm{GHz}$, and the transmission rate of up to $100 \mathrm{Mbps}$, very impressive.

ZigBee is based on IEEE802.15.4 standard developed a short-range, low-rate wireless network communication protocol. 802.15.4 mainly defines the physical layer and link layer specification for short-range communication, while ZigBee mainly defines the specifications of the network layer, the transport layer and the application layer above it.

ZigBee provides a uniform standard for low-speed interconnection between different devices within an individual or household. To form a cheap, low-power and short-range wireless network, provides a convenient way to networking. At the same time, ZigBee can also provide highly reliable wireless data transmission and communication distance can be tens of meters to several hundred meters or even a few kilometers, and supports unlimited expansion.

Each wireless communication technology has its own advantages and specific to the actual application of these features should be combined to measure. First of all, combined with the actual needs of the home environment, respectively, these various technologies were analyzed in order to find a more suitable home environment networking technology. Bluetooth technology has been mature applications, but the number of nodes connected only 7 or so, this greatly limits the functionality of the home network. Infrared technology security can only be transmitted by close to ensure the accuracy of data transmission, highly susceptible to external interference and it is not suitable for home networking. Radio RF mainly located in the home wireless local area network, can not meet the remote data acquisition and monitoring of the home network needs. Wi-Fi is more suitable for public places to provide free wireless access, and home wireless network is quite different. The Zigbee technology, its low power consumption, high security and self-organization and other advantages, fully meet the needs of home environment, so this paper uses Zigbee technology to form a smart home environment, wireless sensor network.

\section{General Analysis of Zigbee Intelligent Home System}

It focuses on building smart home systems, Internet of things technology in the home life of the concentrated application, so that the owners fully feel the future of home life safety, intelligence, convenience and comfort. In the design process should follow the following principles: (1) feasibility and practicality. At the technical level, the system should ensure its feasibility and economy and the system in the construction process should pay attention to its application and effectiveness. (2) Advanced and mature. In the design process not only to achieve the basic functions, but also should reflect the advanced level, should also be far-sighted, to ensure that the system after many years of dominance. (3) Openness and standards. Currently restricting the development of smart home is a major bottleneck of the system and equipment standards. So in the design of the system should also take into account its openness and standards. (4) Reliability and stability. System reliability and stability of the run-time can not be ignored is an important factor.

Coordinator, terminal node, router is ZigBee wireless network of the three components in the design of these three parts require different. The capacity of the ROM, the coordinator of the largest requirements, because it is the formation of network nodes; and the end nodes, it is the smallest ROM requirements. As a network node, the hardware requirements, the various devices are different, such as RFD only ZigBee network standard to achieve a part of the function, but the FFD 
has achieved its provisions in all the features. Therefore, we choose the home network coordinator of the hardware device, we must choose the FFD device, so you can control and manage the entire home network. In the whole process of setting up the home network, we first set a PAN identifier, and then other sub-devices can be added to the device; followed on the router device selection, we also use the FFD device.

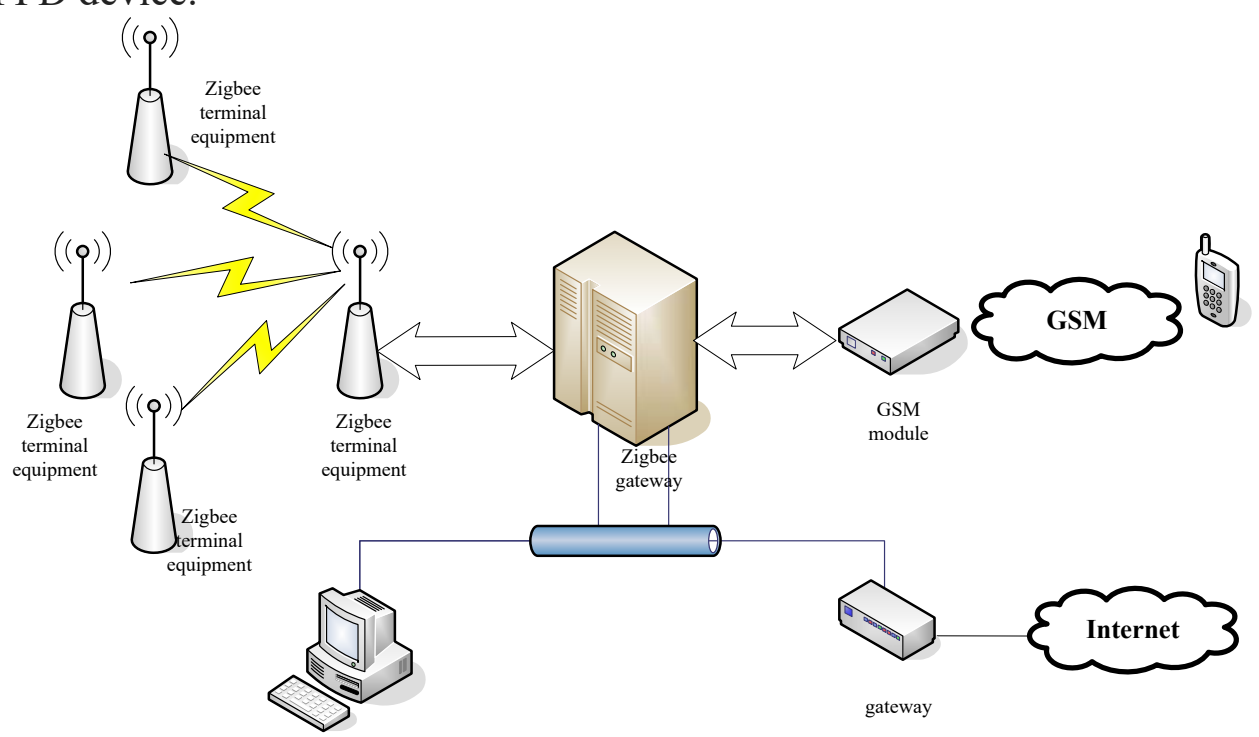

Fig. 1 Zigbee Smart Home System

\section{Hardware Node Design of Intelligent Home System}

In the ZigBee network coordinator is the core, the communication between nodes through it to be responsible. Usually the coordinator node and the end node are controlled by the microcontroller (MCU) and are connected to the ZigBee transceiver via a serial peripheral interface (SPI). SPI is a high-speed, full-duplex, synchronous communication bus and the communication principle is simple to master-slave work. In the ZigBee node, MCU is the master of SPI communication, CC2420 RF module as a slave device. SPI is also a serial communication protocol and the data is a one-bit transmission. The advantage of the transmission mode is that the SPI allows data to be transmitted one bit at a time, and even allows pauses, while ordinary serial communications transfer at least eight bits of data at a time.

Wireless communication module (CC2420 RF module), serial port transceiver module (RS232 serial port), microprocessor module (PIC18LF4620 MCU) and power module are the hardware modules of home wireless sensor network coordinator node, as shown in Figure 5-1. Wireless communication module is mainly responsible for wireless communication with each node, exchange and send and receive data collection; serial module is mainly responsible for receiving instructions sent home server and home server to send the various functions of node information; microprocessing is mainly used to deal with itself and is Other endpoint data transmission, and as far as possible to achieve safe and reliable communication; power module is to ensure that all departments of the system can have the normal operation of energy.

Terminal node design in the microcontroller and RF chip selection and design of the external circuit, and the coordinator node should remain highly consistent. Terminal node is to achieve specific functions in the network, so each terminal node design corresponds to a specific application.

\section{Software Design of Intelligent Home System}

The main program consists of three parts: the protocol stack section, application source files and configuration source files (which can be used to define the node functions and types, etc.), other necessary files. 
According to the function, the system mainly by the network coordinator module, the terminal equipment module as well as the network management system three major constitutions, the concrete function as well as realizes following. ZigBee.def can be achieved through the definition of network coordinator work. Under normal circumstances, the definition of the node, including the 64-bit network address, the use of which frequency band, what specific functions, what type, the transmission power is how much, choose which channel.

The terminal node can be defined by executing the file ZigBee.def. ZigBee.def file can be defined on the number of nodes, such as the node's network address, type, function, transmit power, the use of frequency and channel selection. RFD nodes also have a sleep mode of work, because the coordinator can not determine the RFD node (such as the RFD node), the RFD node can not determine the RFD node, When it is in the wake-up state, it is not possible to send data directly to the RFD node. If the RFD node is awakened, the RFD node sends the primitive MLME-SYNC. Request to achieve the request synchronization with the coordinator, and requests the network coordinator for its own cache of the frame.

\section{Conclusion}

In this paper, based on wireless sensor network technology, intelligent home network can monitor the home environment, network convenience, but also embedded in the wireless sensor furniture, household appliances and other daily necessities connected with the Internet, to achieve long-range control. Home network provides us with a comfortable, convenient and humane intelligent home environment.

\section{References}

[1] K. Sohrabi, et al. Protocols for Self-Organization of a Wireless Sensor Network [J]. IEEE Pers. Commune, 2011, 10, 15-24

[2] Ramesh P Rao, Niranjan Tripathy and William P Dukes. Dealer Bid-Ask Sreads and Options Trading on OTC Stocks [J].The Journal of Financial Research, 2011（4）:317-325

[3] Christopher G. Lamoureux and Gary C. Sanger. Firm Size and Turn of the year Effects in the OTC/NASDAQ Market [J]. The Journal of Finance, 2010 (5):1219-1245

[4] Kenneth K. Leong and Janis K. Zaima. Further Evidence of the Small Firm Effect: A Comparison of NYSE-AMEX and OTC Stocks [J]. Journal of Business Finance \& Accounting,1991 (1) : 117-124

[5] Niranjan Tripathy, Ramesh P. Rao. Adverse Selection, Spread Behavior and Over-The-Counter Seasoned Equity Offerings [J]. The Journal of Financial Research, 2002 (1) : 39-56 